\title{
Abstract Classes with few models have 'homogeneous-universal' models
}

\author{
J. Baldwin * \\ Department of Mathematics \\ University of Illinois, Chicago \\ S. Shelah Department of Mathematics \\ Hebrew University of Jerusalem ${ }^{\dagger}$
}

August 9, 2018

This paper is concerned with a class $\mathbf{K}$ of models and an abstract notion of submodel $\leq$. Experience in first order model theory has shown the desirability of finding a 'monster model' to serve as a universal domain for $\mathbf{K}$. In the original constructions of Jónsson and Fraissè, $\mathbf{K}$ was a universal class and ordinary substructure played the role of $\leq$. Working with a cardinal $\lambda$ satisfying $\lambda^{<\lambda}=\lambda$ guarantees appropriate downward Lowenheim-Skolem theorems; the existence and uniqueness of a homogeneous-universal model appears to depend centrally on the amalgamation property. We make this apparent dependence more precise in this paper.

The major innovation of this paper is the introduction of weaker notion (chain homogeneous-universal) to replace the natural notion of $(\mathbf{K}, \leq)$ homogeneous-universal model. Modulo a weak extension of ZFC (provable if $\mathrm{V}=\mathrm{L}$ ), we show (Corollary 5.24) that a class $\mathbf{K}$ obeying certain minimal restrictions satisfies a fundamental dichotomy: For arbitrarily large $\lambda$, either $\mathbf{K}$ has the maximal number of models in power $\lambda$ or $\mathbf{K}$ has a unique chain

\footnotetext{
*Partially supported by N.S.F. grant 90000139

${ }^{\dagger}$ Both authors thank the U.S. Israel Binational Science foundation for its support of this project. This is item 393 in Shelah's bibliography.
} 
homogenous-universal model of power $\lambda$. We show (5.25) in a class with amalgamation this dichotomy holds for the notion of $\mathbf{K}$-homogeneous-universal model in the more normal sense.

The methods here allow us to improve our earlier results [5] in two other ways: certain requirements on all chains of a given length are replaced by requiring winning strategies in certain games; the notion of a canonically prime model is avoided. A full understanding of these extensions requires consideration of the earlier papers but we summarise them quickly here.

Shelah emphasized in [13] that Tarski's union theorem has two components: closure under unions; each union is an amalgamation base (smoothness). The first is used to show the existence of a homogeneous universal model; the second is needed for uniqueness. In this paper we show that closure can be replaced by the existence of a bound for each chain and even stronger that we need the boundedness only for a 'dense' (in a sense made precise by a game defined below) set of chains.

In [5] we established a dichotomy between the smoothness of a class and a nonstructure theorem. There was a weakness in our result; although the definition of smooth (there is a unique compatibility class over each chain) does not involve the concept of a canonically prime model we only established the theorem for classes equipped with a notion of a canonically prime model. We remedy that difficulty in this paper at some cost. First we require some additional set theoretic hypotheses (all provable if $\mathbf{V}=\mathbf{L}$ ). Second we must weaken the conclusion. Instead of coding stationary sets we can only guarantee that there are $2^{\lambda}$ models of power $\lambda$.

The results here generalize an earlier result proved in [12]. That paper dealt with a class $\mathbf{K}$ satisfying the axioms discussed here but also closed under unions of $\mathbf{K}$-chains and that was smooth. Theorem 3.5 and Claim 3.4 of [12] imply that if $\mathbf{K}$ is categorical in $\lambda$ and has few models of power $\lambda^{+}$ then the unique model of power $\lambda$ is an amalgamation base.

We rely on many notations and definitions from [4] and [5] but only on rudimentary results from those papers.

Section a contains the background notation. In Section of this paper we introduce several games; we are able to express questions about the smoothness or boundedness of a class $\mathbf{K}$ in terms of winning strategies for these games. Section 3 describes the set theoretic hypotheses necessary for our construction. We show in Section 4 that a winning strategy for Player NAM in Game $2(\lambda, \kappa)$ implies the existence of many models. Section 5 
translates the existence of a winning strategy for a player (B) trying to show chains are bounded and the failure of the player trying to force nonamalgamation (NAM) to have a winning strategy into the existence and, if there are few models, the uniqueness of 'chain homogeneous-universal' models. For a class with amalgamation this yields uniqueness and existence of the $(\mathbf{K}, \leq)$ homogeneous-universal models. In Section 6 we summarise our results and suggest some open problems. Section 7 contains proofs of the combinatorial results summarised in Section 3 .

We thank Chris Laskowski and Bradd Hart for their valuable advice in preparing this paper.

\section{Setting the Scene}

Most of the notions used in this paper are defined in [4] or [5]. They or minor variants occur in earlier papers of Shelah, specifically [13.

$(\mathbf{K}, \leq)$ is an abstract class satisfying Axiom group A of [4]:

A0 If $M \in K$ then $M \leq M$.

A1 If $M \leq N$ then $M$ is a substructure of $N$

A2 $\leq$ is transitive.

A3 If $M_{0} \subseteq M_{1} \subseteq N, M_{0} \leq N$ and $M_{1} \leq N$ then $M_{0} \leq M_{1}$.

We review here some of the less common concepts. All notions defined with cardinal parameters have the obvious variants obtained by, e.g., replacing $\lambda$ by $<\lambda . \quad \mathbf{K}_{\lambda}$ is the class of members of $\mathbf{K}$ with cardinality $\lambda . \mathrm{A}(<\lambda, \kappa)$ chain is a $\mathbf{K}$-increasing chain of cofinality $\kappa$ members of $\mathbf{K}(i<j$ implies $M_{i} \leq M_{j}$ ), each of cardinality $<\lambda$. A chain $\underline{M}$ is $\mathbf{K}$-bounded if there is an $M \in \mathbf{K}$ and a compatible family of maps $f_{i}$ mapping $M_{i}$ into $M$. $\mathbf{K}$ is $(<\lambda, \kappa)$-bounded if each $(<\lambda, \kappa)$-chain is bounded. $\mathbf{K}$ is $(<\lambda,<\kappa)$-closed if the union of each such chain is in $\mathbf{K}$. Sections 2 and 4 of [5] contain a number of examples that illustrate these concepts.

1.1 Assumptions. We fix for this paper a cardinal $\lambda$ with the following properties.

i) $\lambda$ is a regular cardinal greater than the size of the vocabulary of $\mathbf{K}$. 
ii) There are no maximal models in $\mathbf{K}_{<\lambda}$.

iii) $\mathbf{K}$ is $(<\lambda, \lambda)$-closed.

iv) The $<\lambda$-Löwenheim Skolem property holds.

v) For some regular $\kappa<\lambda$, some $S \subseteq \lambda$, and cardinal $R \leq \lambda, \bigotimes_{\lambda, \kappa, R}(S)$ holds.

We briefly justify this group of assumptions. Property $\varangle$ is explained in Section 3. If for some $\kappa$ and $\lambda, \bigotimes_{\lambda, \kappa, R}(S)$ holds then in particular $\diamond(\lambda)$ holds and so $\lambda^{<\lambda}=\lambda$. Thus, there will be no cardinality obstruction to obtaining homogenous-universal models of power $\lambda$. Assuming $\mathbf{K}$ is $(<\lambda, \lambda)$ closed is virtually a convention. If we dropped that hypothesis we would reach the conclusion that $2^{\lambda}$ structures of power $\lambda$ were increasing unions of members of $\mathbf{K}_{<\lambda}$. We say $\mathbf{K}$ has few models if there are $<2^{\lambda}$ isomorphism types of models of power $\lambda$. We will need to assume a boundedness hypothesis on $\mathbf{K}$; but it varies with particular theorems in this paper. We fix the proper $\kappa, R$ and $\lambda$ in clause $\mathrm{v}$ ) at the appropriate time.

\section{Two Games}

This paper is crucially concerned with the question of exactly how one bounds an increasing $\mathbf{K}$-chain of models. We have learned that it is not essential to posit that all chains are bounded; only a sufficient number of them. This sufficiency can be described in terms of winning strategies for certain games. Game $1(\lambda, \kappa)$ is played between player $\mathrm{B}$ who wants to establish that $\mathbf{K}$ is $(<\lambda, \leq \kappa)$-bounded and player NB who is trying to show the opposite.

2.1 Definition. i) For an ordinal $\alpha$, a play of Game $1(\lambda, \alpha)$ lasts at most $\alpha$ moves. During the play Player B chooses models $\left\langle L_{i}: i<\delta \leq \alpha\right\rangle$; Player NB chooses models $\left\langle P_{i}: i<\delta \leq \alpha\right\rangle$. At move $\beta$,

(a) Player B chooses a model $L_{\beta}$ in $\mathbf{K}_{<\lambda}$ that is a proper $\mathbf{K}$-extension of all the structures $P_{\gamma}$ for $\gamma<\beta$.

(b) Player NB chooses a model $P_{\beta}$ in $\mathbf{K}_{<\lambda}$ that is a proper $\mathbf{K}$-extension of $L_{\beta}$. 
Either player loses the game if at some stage he does not have a legal move. Player B wins if $\underline{P}$ is bounded; otherwise Player NB wins. Player $\mathrm{B}$ has the advantage of playing first at limit ordinals; the price is that he must guarantee the existence of a bound at each limit stage.

ii) We say Player B has a winning strategy for Game $1(\lambda,<R)$ if he has a uniform strategy to win all plays of Game $1(\lambda, \mu)$ for each ordinal $\mu<R$.

2.2 Proposition. If $\mathbf{K}$ is $(<\lambda, \leq \kappa)$-bounded then Player $B$ has a winning strategy for Game $1(\lambda, \kappa)$.

We continue to use the notations for properties of embeddings of chains established in [5] and briefly reviewed in Section 11.

2.3 Definition. i) The $\mathbf{K}$-increasing chain $\underline{N}$ of members of $\mathbf{K}_{<\lambda}$ extends $\underline{N^{\prime}}$ if $\underline{N^{\prime}}$ is an initial segment of $\underline{N}$

ii) Two extensions $\underline{N}^{\prime}$ and $\underline{N}^{\prime \prime}$ of a chain $\underline{N}$ can be amalgamated over $\underline{N}$ if there is an $N \in \mathbf{K}$ and embeddings of $\underline{N}^{\prime}$ and $\underline{N}^{\prime \prime}$ into $N$ which agree on $\underline{N}$.

iii) The $\mathbf{K}$-increasing chain $\underline{N}$ of members of $\mathbf{K}_{<\lambda}$ is a $\lambda$-amalgamation base if $\underline{N}$ is bounded and every pair of $\mathbf{K}$-extensions of $\underline{N}$, each in $\mathbf{K}_{<\lambda}$, can be amalgamated over $\underline{N}$ in $\mathbf{K}_{<\lambda}$.

To say $\underline{N}$ is an amalgamation base is to say $\mathbf{K}$ is smooth at the chain $\underline{N}$; if $\mathbf{K}$ satisfies the $<\lambda$-Löwenheim Skolem property the increasing chain $\underline{N}$ of members of $\mathbf{K}_{<\lambda}$ is a $\lambda$-amalgamation base if and only if all extensions of $\underline{N}$ are compatible over $\underline{N}$.

A canonically prime model over a chain $\underline{M}$ can be viewed as a 'strategy', choosing, at each limit stage, one among a number of possible compatibility classes over an initial segment of the chain. At stage $\delta$, the strategy depends not on the actual ordinal $\delta$ but on the isomorphism type of $\underline{M} \mid \delta$. Game 2 concerns the construction of chains that are amalgamation bases. We rename Player B as Player NAM (he wants to prevent amalgamation) and Player NB as Player AM (he wants to build an amalgamation base).

2.4 Definition. i) A play of Game $2(\lambda, \alpha)$ lasts $\leq \alpha$ moves. The play of the game is exactly as in Game 1 (with NAM replacing B, AM replacing 
NB). A player who has no legal move loses; however, Player NAM wins instantly if for some limit ordinal $\beta \leq \alpha, \underline{P} \mid \beta$ is bounded but is not a $\lambda$-amalgamation base.

ii) Game $2(\lambda,<\beta)$ is defined similarly, but Player NAM wins only if for some $\alpha<\beta, \underline{P} \mid \alpha$ is bounded but is not a $\lambda$-amalgamation base.

Note that if player NAM wins instantly at some stage then the length of the game is less than $\alpha$.

In both games the decision about a completed game is based only on the second player's moves (NB or AM); apparently to win the first player must force the second to make mistakes. The following lemma shows this intuition is misleading.

2.5 Lemma. Player B (NAM) wins Game 1 (Game 2) $(\lambda, \kappa)$ if and only if the sequence $\bar{L}$ constructed during the play is bounded (and is not an amalgamation base).

Proof. A chain is bounded (an amalgamation base) if and only if each cofinal subsequence is.

The preceding remark is quite straightforward; contrast it with the difficulties involved in considering canonically prime models over subsequences [5].

Note that if player NAM plays a winning strategy for Game 2, he also playing a winning strategy for Game 1.

\section{$3 \otimes$}

We discuss in this section the principle $\varangle$ from [3]. It is a combination of Jensen's combinatorial principles $\square$ and $\diamond$ that will be used in our main construction. Some justification is necessary for the use of such strong set theory. On the one hand, arguments with a strong set theoretic hypothesis that conclude the existence of many nonisomorphic models show it is impossible to prove in ZFC that $\mathbf{K}$ has few models. Thus any 'structure theory' that could be established for $\mathbf{K}$ in ZFC would have to allow the maximal number of models in a class with 'structure'. On the other, while for ease of statement we assert that these combinatorial principles follows from $\mathrm{V}=\mathrm{L}$, in fact they only depend on the structure of the subsets of $\lambda$. Thus $2^{\lambda}$ can 
be as large as desired while keeping $\nabla_{\lambda, \kappa, R}$ for each $\kappa<\lambda$ and some $R \leq \lambda$. Moreover, the consistency of $\varangle$ can be obtained by a forcing extension as well as by an inner model. Some instances of depend only on appropriate instances of $\mathrm{GCH}$. We begin by establishing some notation.

3.1 Notation. i) For any set of ordinals $C$, acc $[C]$ denotes the set of accumulation points of $C$, i.e., the $\delta \in C$ with $\delta=\sup C \cap \delta$. nacc $[C]$ denotes $C-\operatorname{acc}[C]$.

ii) Fix regular cardinals $\lambda>\kappa$; let $C^{\kappa}(S)$ denote the set of $\delta \in S$ which have cofinality $\kappa$. In the following $\delta$ always denotes a limit ordinal.

iii) Suppose there is $S \subseteq \lambda$ and a collection $\left\langle C_{\delta}: \delta \in S\right\rangle$. Then for any $S_{1} \subseteq S, \tilde{S}_{1}$ denotes $\bar{S}_{1} \cup \cup\left\{C_{\delta}: \delta \in S_{1}\right\}$.

3.2 Definition. $\left[\otimes_{\lambda, \kappa, R}(S)\right]$ We say that $\mathcal{C}=\left\langle C_{\alpha}: \alpha \in S\right\rangle$ and the sequence $\mathcal{A}=\left\langle A_{\alpha}: \alpha<\lambda\right\rangle$ witness that $\lambda, \kappa, R$ satisfy $\left[\otimes_{\lambda, \kappa, R}(S)\right]$ if for some subset $S_{1}$ of $S$ the following conditions hold.

i) $\kappa$ is a regular cardinal $<\lambda, R$ is an ordinal $\leq \lambda$.

ii) $S$ is stationary in $\lambda$ and contains all limit $\delta \in \lambda$ with $\operatorname{cf}(\delta)<R$; S contains only even ordinals.

iii) $S_{1} \subseteq C^{\kappa}(S)$.

iv) Each $C_{\delta} \subseteq S$.

v) If $\alpha \in S$

(a) $C_{\alpha}$ is a closed subset of $\alpha$,

(b) if $\beta \in C_{\alpha}$ then $C_{\beta}=C_{\alpha} \cap \beta$,

(c) $\operatorname{otp}\left(C_{\alpha}\right) \leq \max (R, \kappa)$, more precisely,

1. $\operatorname{otp}\left(C_{\alpha}\right)=\kappa$ if $\alpha \in S_{1}$ and

2. $C_{\alpha} \cap \tilde{S}_{1}=\emptyset$ and $\operatorname{otp}\left(C_{\alpha}\right)<R$ if $\alpha \in S-\tilde{S}_{1}$ is a limit ordinal,

(d) all nonaccumulation points of $C_{\alpha}$ are even successor ordinals.

vi) If $\delta \in S$ is a limit ordinal then $C_{\delta}$ is a club in $\delta$. 
vii) Each $A_{\alpha} \subseteq \alpha$ and

(a) if $\beta \in S_{1}$ and $\alpha \in \operatorname{acc}\left[C_{\beta}\right]$ then $A_{\alpha}=A_{\beta} \cap \alpha$,

(b) for $A \subseteq \lambda$ and any closed unbounded subset $E$ of $\lambda, X_{E}=\{\delta \in$ $\left.S_{1}: \delta \in E \& \operatorname{acc}\left[C_{\delta}\right] \subseteq E \& A_{\delta}=A \cap \delta\right\}$ is stationary in $\lambda$.

3.3 Definition. $\bigotimes_{\lambda, \kappa, R}$ holds if for some subset $S \subseteq \lambda, \bigotimes_{\lambda, \kappa, R}(S)$ holds.

We discuss the truth of this proposition for various choices of $\lambda$ and $\kappa$. We begin with a case proved in ZFC; some cases of the GCH are needed to find cardinals satisfying the hypotheses. Combining the methods of [3], [11], and [8] yields the following result; we include a full proof in an appendix at the suggestion of the referee.

3.4 Lemma. Suppose $\mu^{\kappa}=\mu$ and $2^{\mu}=\mu^{+}=\lambda$. If $S^{*} \subseteq S^{\kappa}(\lambda)$ is stationary then there is an $S \subseteq \lambda$ such that $\otimes_{\lambda, \kappa, \omega}$ holds and $S \cap C^{\kappa}(\lambda) \subseteq S^{*}$.

This combinatorial result is sufficient for our model theoretic constructions if the problematical model theoretic situation concerns chains of length $\omega(\kappa=\omega$. To deal with chains of longer length (this is essential; see [5]), the following stronger combinatorial principal is needed.

Lemma $3.5(\mathbf{V}=\mathbf{L})$. If $\lambda=\mu^{+}$and $\kappa \leq \mu$, then for some stationary $S$, $\otimes_{\lambda, \kappa, \lambda}(S)$.

\section{Players B and NAM construct many mod- els}

We show in this section that if for some $\kappa<\lambda$ and an ordinal $R \leq \lambda$ Player B has a winning strategy for Game $1(\lambda, \kappa)$ and Game $1(\lambda,<R)$, $\mathbf{K}$ is $(\lambda, \geq R$ )-bounded, and Player NAM has a winning strategy for Game $2(<\lambda, \kappa)$ then $\mathbf{K}$ has the maximal number of models in power $\lambda$. This is of course a technically weaker conclusion than in [5] where we showed that if $\mathbf{K}$ is not smooth then $\mathbf{K}$ codes stationary sets. But we have weaker

hypotheses here and this conclusion expresses a somewhat weaker intuition of nonstructure. This weakening of the result is reflected in a complication of the main argument. In the earlier paper we constructed for each stationary set $W$ 
a model $M^{W}$ coding $W$. Here, we construct $2^{\lambda}$ models simultaneously and destroy putative isomorphisms between them enroute. The second author has in mind a more elaborate version of our construction, which we don't expand on here, that recovers the coding of stationary sets in this context. The stronger conclusion in [5] assumed the class $\mathbf{K}$ was equipped with notions of free amalgamation and canonically prime models; here we have no such assumption.

The many-models proof given here illustrates the role of canonically prime model notion. When cpr is in the formal metalanguage a particular choice of limit model is specified so one can construct a sequence of models and code a stationary set by asking the question, 'Is the limit model at $\delta$ the canonically prime model?' When we remove this notion from the formal language we have to destroy isomorphisms between the possible choices of a limit model. Diamond allows us to do this.

We thank Bradd Hart for suggesting a simplification in the proof of the main result.

4.1 Theorem. Fix regular cardinals $\kappa<\lambda$ and an ordinal $R \leq \lambda$ satisfying $\otimes_{\lambda, \kappa, R}$. Suppose

i) $\mathbf{K}$ is $(<\lambda, \geq R)$-bounded; Player $B$ has a winning strategy for Game 1 $(\lambda,<R)$.

ii) Player NAM has a winning strategy for Game $2(\lambda, \kappa)$.

iii) Player $B$ has a winning strategy for Game $1(\lambda, \kappa)$.

Then there are $2^{\lambda}$ members of $\mathbf{K}$ with cardinality $\lambda$. Moreover, these models are mutually $\leq_{\mathbf{K}}$-non-embeddible.

4.2 Remark. The connection between hypotheses i) and iii) deserves some comment. If $\kappa>R$ then iii) implies the second clause of $\mathrm{i}$ ). It is tempting to think that i) implies iii). However, if $\kappa>R$ a game of length $>\kappa$ might still have cofinality $<R$. Neither clause of i) guarantees a winning stategy for that game.

Proof. Fix $S,\left\langle C_{i}: i \in S\right\rangle$ and $\left\langle A_{\alpha}: \alpha<\lambda\right\rangle$ and $S_{1}$ to witness $\otimes_{\kappa, \lambda, R}$.

Using a pairing function and condition vii) of Definition 3.2 we can find $\left\langle\nu_{\alpha}, \eta_{\alpha}, f_{\alpha}: \alpha<\lambda\right\rangle$ with $\nu_{\alpha}, \eta_{\alpha}$ in $2^{\alpha}$ and $f_{\alpha}$ a function from $\alpha$ to $\alpha$ such that 
for any $\nu, \eta \in \lambda$, any function $f: \lambda \mapsto \lambda$, and any closed unbounded subset $E$ of $\lambda$, for some $\alpha \in S_{1} \cap E$, acc $\left[C_{\alpha}\right] \subseteq E$, and for every $\beta \in\{\alpha\} \cup \operatorname{acc}\left[C_{\alpha}\right]$ we have $\nu_{\beta}=\nu \cap \beta, \eta_{\beta}=\eta \cap \beta, f$ restricted to $\beta$ is $f_{\beta}$.

By induction, for each $\alpha<\lambda$ and each $\nu \in 2^{\alpha}$ we define a structure $N_{\nu}$ whose universe is an ordinal $<\lambda$ so that if $\alpha<\gamma, \nu \in 2^{\alpha}, \eta \in 2^{\gamma} N_{\nu}$ is a proper $\mathbf{K}$-submodel of $N_{\eta}$. Then we finish the construction by defining for each $\eta \in 2^{\lambda}, N_{\eta}$ as $\cup_{\alpha<\lambda} N_{\eta \mid \alpha}$.

When $\eta \in 2^{\alpha}$, we write $\underline{N}_{\eta}$ for the sequence $\left\langle N_{\eta \mid \beta}: \beta<\alpha\right\}$.

We now define a winning strategy against the restriction of a play of a game to a club that is appropriate for the arguments here.

4.3 Definition. Fix $\alpha \in S$. Suppose $\underline{N}=\left\langle N_{i}: i<\alpha\right\rangle$ is a $\mathbf{K}$-increasing chain and $C$ is a club in $\alpha$ such that nacc $[C]$ is a set of even successor ordinals. Consider an initial segment of a play of Game 1 (Game 2) where $L_{i}=N_{\gamma_{i}}$, $P_{i}=N_{\gamma_{i+1}-1}$, and $\left\langle\gamma_{i}: i<\alpha_{0}\right\rangle$ is an enumeration of $C$. If $\alpha$ is a successor and $\alpha-1 \in C$, say $C=\left\{\gamma_{i}: i \leq \alpha_{0}\right\}$ then $P_{\alpha_{0}}$ is not defined by the preceding; let $P_{\alpha_{0}}=N_{\alpha-1}$. (Otherwise, $P_{i}$ is well defined since by Definition $3.2 \mathrm{v}$ ) d) $\gamma_{i+1}$ is a successor ordinal. Note $\gamma_{i+1}-1 \neq \gamma_{i}$ because $C$ contains only even ordinals.)

Suppose that each $L_{i}$ has been chosen by Player B (NAM)'s winning strategy for this game and suppose $N_{\alpha}$ is now chosen according to Player B (NAM)'s winning strategy in this play of the game. We say $N_{\alpha}$ has been chosen by playing Player B (NAM)'s winning strategy for Game 1 (Game 2) on $\underline{N} \mid C$.

The notation $\underline{N} \mid C$ is slightly inaccurate since when $\alpha$ is a successor the choice depends on $N_{\alpha-1}$. Nevertheless we adopt the notation because of its suggestiveness in the limit ordinal case.

Note that if $\beta \in C_{\alpha}$ then playing according to the winning strategy on $\underline{N} \mid C_{\beta}$ is by condition of v) b of Definition 3.2, the same as playing a winning strategy on an initial segment of $\underline{N} \mid C_{\alpha}$.

We use the following ad hoc notation. We need to introduce this notation because we are using winning strategies in both games 1 and 2 as the hypothesis for the construction. If we simply assumed $\mathbf{K}$ is $(\lambda, \lambda)$-bounded then we wouldn't need this curlicue.

4.4 Notation. For $\alpha<\beta \in S, C_{\beta, \alpha}$ denotes $\left\{\epsilon: \epsilon \in C_{\beta} \& \epsilon \geq \alpha\right\}$. 
4.5 Construction. We split the construction into several cases. Let

$$
\tilde{S}_{1}=S_{1} \cup \cup\left\{C_{\delta}: \delta \in S_{1}\right\}
$$

Most cases in the construction are defined by playing the winning strategy of Player B or Player NAM on a closed unbounded subset of $\beta$. Condition v) of $\nabla_{\kappa, \lambda, R}(S)$ guarantees that the various cases cohere. Certain inductive properties of the construction are incorporated in the description of the cases.

At stage $\beta$, we have fixed $\nu, \eta \in 2^{\beta}$ and a map $f_{\beta}$ from $\beta$ to $\beta$. For each $\tau \in 2^{\beta}$ we construct a model $N_{\tau}$. In many cases $N_{\tau}$ is chosen by playing the winning strategy for Player B or player NAM on $\underline{N}_{\tau} \mid C_{\beta}$. To see that these strategies do not conflict note first that if every play on $\underline{N}_{\tau} \mid C_{\beta}$ has been played by the winning strategy in either game then it has been played according to a winning strategy for Game 1 (as the winning strategy for Game 2 also wins game 1) and so inductively $N_{\tau}$ can be chosen by Player B's winning strategy in Game 1. Moreover, since Player B has a uniform winning strategy for all games of length less than $\lambda$ his play does not depend on the particular game of length less than $\lambda$ that is being considered. The inductive hypothesis in the cases where Player NAM's winning strategy in Game 2 is used are verified below. The key step in the proof is subcase b) of Case IV. The other stages are preserving the induction hypothesis.

Case I. $\beta$ is a successor ordinal $\gamma+1$. We will choose $N_{\tau}$ as a proper $\mathbf{K}$ extension of $N_{\tau \mid \beta}$. This is possible since we have assumed that there are no maximal models in $\mathbf{K}_{<\lambda}$. In certain subcases however we must be more specific.

Subcase a. $\beta \in S-\tilde{S}_{1}$. Choose $N_{\tau}$ by playing Player B's winning strategy for Game 1 on $\underline{N}_{\tau} \mid C_{\beta}$.

Subcase b. $\beta \in \tilde{S}_{1}$. If Player NAM has already won Game 2 played on $\underline{N}_{\tau} \mid C_{\beta}$. at some stage $\gamma<\beta$, play the winning strategy for Player B on $C_{\beta, \gamma}$. If not, $\underline{N}_{\tau} \mid C_{\beta}$. has been played by the winning strategy of Player NAM. Choose $N_{\tau}$ by playing the winning strategy for Player NAM for Game 2 on $\underline{N}_{\tau} \mid C_{\beta}$.

Case II. $\beta$ is a limit ordinal and $\beta \notin S$. By ii) of Definition 3.2, $\beta$ has cofinality at least $R$. Choose $N_{\tau}$ to bound $\underline{N} \mid \tau$ (by $(<\lambda, \geq R$ ) boundedness) and with $\left|N_{\tau}\right|<\lambda$ (by the $<\lambda$-Löwenheim Skolem property). 
Case III. $\beta$ is a limit ordinal and $\beta \in\left(S-\tilde{S}_{1}\right)$. Since $\operatorname{cf}\left(C_{\beta}\right)<R$, we can choose $N_{\tau}$ to bound $\underline{N}_{\tau} \mid \beta$, by playing Player B's winning strategy for Game 1 on $\underline{N}_{\tau} \mid C_{\beta}$. (Note $C_{\beta}$ is unbounded in $\beta$ by Definition 3.2 vi).

Case IV. $\beta \in \tilde{S}_{1} ; \beta$ a limit ordinal. The situation is interesting only if $\beta=\cup \underline{N}_{\nu}=\cup \underline{N}_{\eta}, f_{\beta} \mid N_{\nu \mid \gamma}$ is a $\mathbf{K}$-embedding for each $\gamma<\beta$ and $\tau=\nu$. Unless all of these conditions hold, choose $N_{\tau}$ as in Case Ib) by playing NAM's strategy on $\underline{N}_{\tau} \mid C_{\beta}$ or B's winning stategy on $\underline{N}_{\tau} \mid C_{\beta, \gamma}$. If they do hold there are two subcases.

Subcase a. $\underline{N}_{\tau}$ is an amalgamation base. If $\gamma \in C_{\beta}$ then by Subcase Ib, or Subcase IV b) at stage $\gamma, N_{\tau \mid \gamma}$ was chosen by Player NAM's winning strategy on $\underline{N}_{\tau} \mid C_{\gamma}$. So, we are able to apply Player NAM's winning strategy for Game $2(\lambda, \kappa)$ on $\underline{N}_{\tau} \mid C_{\beta}$ to choose $N_{\tau}$.

Subcase b. $\underline{N}_{\tau}$ is not an amalgamation base. $N_{\eta}$ has been defined. Thus there are incompatible bounds $A_{1}, A_{2} \in \mathbf{K}_{<\lambda}$ for $\underline{N}_{\tau}$. If there is an extension $\hat{f}_{\beta}$ of $f_{\beta}$ such that $\hat{f}_{\beta}\left(A_{1}\right)$ is compatible with $N_{\eta}, N_{\tau}=A_{2}$; otherwise $N_{\tau}=A_{1}$.

This completes the construction. The various cases are clearly disjoint. We finish the proof by proving the following claim.

Claim. If $\sigma \neq \tau \in 2^{\lambda}$ then there is no $\mathbf{K}$-embedding of $N_{\sigma}$ into $N_{\tau}$.

Suppose for contradiction that $f$ is such an embedding. For $\alpha$ in a closed unbounded subset $C$ of $\lambda$,

$$
\alpha=\cup_{\beta<\alpha} N_{\sigma \mid \beta}=\cup_{\beta<\alpha} N_{\tau \mid \beta} .
$$

and and for each $\beta<\alpha, f \mid N_{\sigma \mid \beta}$ is an $\mathbf{K}$-embedding into $N_{\tau \mid \beta^{\prime}}$ for some $\beta^{\prime}<\alpha$. Thus, there is an $\alpha \in C \cap S_{1}$ with $f_{\alpha}=f\left|\alpha, \nu_{\alpha}=\sigma\right| \alpha, \eta_{\alpha}=\tau \mid \alpha$ and $\operatorname{acc}\left[C_{\alpha}\right] \subseteq C$. Moreover, for $\beta \in \operatorname{acc}\left[C_{\alpha}\right], f \mid \beta=f_{\beta}$. Since $\alpha \in S_{1}$, $\operatorname{cf}(\alpha)=\kappa$. Since Player NAM has a winning strategy in Game $2(\lambda, \kappa)$, either Player NAM wins Game 2 at stage $\alpha$ or Player NAM won at some earlier stage $\delta$. In the first event, subcase IV b) of the construction applied at stage $\alpha$, guarantees there is no embedding of $N_{\nu}=N_{\sigma \mid \alpha}$ into any extension of $N_{\eta}=N_{\tau \mid \alpha}$. In the second event, $\delta \in \operatorname{acc} C_{\alpha}$ so by condition vii) a of Definition $3.2 A_{\delta}=A_{\alpha} \cap \delta$. That is, $f_{\delta}: \delta \mapsto \delta$ and since $f_{\alpha}$ is a (sequence of) $\mathbf{K}$-embedding(s) so is $f_{\delta}$. Since $f_{\delta}=f \mid \delta$, subcase IV b) applied at stage 
$\delta$ guarantees there is no extension of $f \mid \delta$ mapping $N_{\sigma \mid \delta}$ into any extension of $N_{\tau \mid \delta}$ and thus no extension of $f \mid \alpha$ mapping $N_{\sigma \mid \alpha}$ into any extension of $N_{\tau \mid \alpha}$. Thus there is no K-embedding of $N_{\sigma}$ into $N_{\tau}$ and we finish.

\section{Classes with few models have 'homogeneous- universal' models}

We consider here several variants on the notion of homogeneous-universal model and establish the existence and uniqueness of models satisfying one of these notions for a class that has few models.

5.1 Assumption. In this section we assume that the class $\mathbf{K}$ has a notion of strong submodel satisfying the axioms of group A in [4] (listed in Section (1)), the properties of $\lambda$ enumerated in Section 11 and

i) $\mathbf{K}$ has fewer than $2^{\lambda}$ models of power $\lambda$.

ii) $\mathbf{K}$ is $(<\lambda,<\lambda)$-bounded.

All the results of this section go through under these assumptions. Since some of them require slightly less, many of the statements repeat these overriding hypotheses or stipulate more technical conditions that suffice.

The following obvious consequence of Theorem 4.1 is a key to this section.

5.2 Lemma. For a regular cardinal $\kappa<\lambda$ such that some regular $R \leq \lambda, \lambda$ satisfies ${ }_{\lambda, \kappa, R}$, Assumption 5.1 implies that Player NAM does not have a winning strategy for Game $2(\lambda, \kappa)$.

5.3 Refinement. Checking the hypotheses for Theorem 4.1, we see that instead of assuming $\mathbf{K}$ is $(<\lambda,<\lambda)$-bounded it suffices to assume $\mathbf{K}$ is $(<$ $\lambda, \geq R$ )-bounded and Player B has a winning strategy for Game $1(\lambda,<R)$.

Our key idea is to redo the Fraisse-Jonsson construction in a category where models have been replaced by chains that are amalgamation bases. While we can not derive the amalgamation property directly from a 'few models' hypothesis, we are able to derive the existence of a sufficient number of amalgamation bases to carry out the construction. 
5.4 Definition. Let $\mathbf{K}_{<\lambda}^{*}$ be the class of $(<\lambda,<\lambda)$-chains that are amalgamation bases and such that every initial segment of the chain with limit length is an amalgamation base.

We define a partial order on these chains.

5.5 Definition. Let $\underline{M}$ and $\underline{N}$ be $(<\lambda,<\lambda)$-chains.

i) $\underline{M} \sim \underline{N}$ if $\bigcup \underline{M}=\bigcup \underline{N}$ and each $M_{i}$ is $\mathbf{K}$-embedded in some $N_{j}$ by the identity and vice versa.

ii) $\underline{M} \prec \underline{N}$ if $\underline{M} \sim \underline{N}$ or there is an $N_{i}$ in the sequence $\underline{N}$ such that for each $j$ with $M_{j} \in \underline{M}, M_{j} \leq N_{i}$. (In the latter case, we say $\underline{N}$ properly extends $\underline{M}$.)

5.6 Lemma. i) $\mathbf{K}_{<\lambda}^{*}$ is not empty. Indeed, for each $N \in \mathbf{K}_{<\lambda}$, there is an $\underline{N} \in \mathbf{K}_{<\lambda}^{*}$ with $N_{0}=N$.

ii) For each $(<\lambda,<\lambda)$-chain $\underline{M}$ there is an $\underline{N} \in \mathbf{K}_{<\lambda}^{*}$ that properly extends $\underline{M}$.

Proof. Consider any play of Game $2(\lambda, \kappa)$ where Player NAM chooses $N$ as $L_{1}$. If Player NAM wins each such play then he has a winning strategy for Game $2(\lambda, \kappa)$ played in the class of $\mathbf{K}$-extensions of $N$. By Lemma 5.2, this contradicts Assumption 5.1 i). If not, $\underline{N}=\left\langle N, P_{0}, P_{1}, \ldots\right\rangle$ is the required member of $\mathbf{K}_{<\lambda}^{*}$. For the second claim, apply this argument to a bound for $\underline{N}$.

Now we can regard this collection of chains under this partial order, $\left(\mathbf{K}_{<\lambda}^{*}, \prec\right)$, analogously to our basic notion of an abstract class. This class is easily seen to have the amalgamation property.

5.7 Lemma. If $\underline{M}^{0} \prec \underline{M}^{1}$ and $\underline{M}^{0} \prec \underline{M}^{2}$ and each $\underline{M}^{i}$ is in $\mathbf{K}_{\lambda}^{*}$ then there is a model $N \in \mathbf{K}$ such that both $\underline{M}^{1}$ and $\underline{M}^{2}$ can be embedded in $N$ over $\underline{M}^{0}$.

Proof. Let $M^{1}$ be a bound of $\underline{M}^{1}$ and $M^{2}$ a bound for $\underline{M}^{2}$. Since $\underline{M}^{0}$ is a $\lambda$-amalgamation base these two models and, a fortiori, the sequences can be amalgamated over $\underline{M}^{0}$. 
Note that if $\underline{M}$ is $\mathbf{K}$-embedded in both $\underline{N}$ and $N$, then $\underline{N}$ and $N$ can be amalgamated over $\underline{M}$. Since each element of $\mathbf{K}_{<\lambda}^{*}$ is an amalgamation base the joint embedding property for members of $\mathbf{K}_{<\lambda}^{*}$ is an equivalence relation. Formally

5.8 Definition. Let $\underline{M}, \underline{N} \in \mathbf{K}_{<\lambda}^{*}$. Then $\mathcal{E}(\underline{M}, \underline{N})$ if there exists an $N$ such that both $\underline{M}$ and $\underline{N}$ can be $\mathbf{K}$-embedded in $N$.

To avoid the notational inconvenience of dealing with compatibility classes we posit:

5.9 Assumption. $\mathbf{K}_{<\lambda}^{*}$ has the joint embedding property. I.e., For any $\underline{M}, \underline{N} \in \mathbf{K}_{<\lambda}^{*}$, there exists an $N$ such that both $\underline{M}$ and $\underline{N}$ can be $\mathbf{K}$-embedded in $N$.

We consider four variants on the notion of homogeneous universal. First we list two variants on the normal notion; then we describe the analogous versions for $\mathbf{K}_{<\lambda}^{*}$. First we fix the meanings of universal.

\subsection{Definition.}

i) For any class $\mathbf{K}, M$ is $\mathbf{K}_{<\lambda}$-universal if all members of $\mathbf{K}$ with cardinality less than $\lambda$ can be $\mathbf{K}$-embedded in $M$.

ii) $M$ is $\mathbf{K}_{<\lambda}^{*}$-universal if each member of $\mathbf{K}_{<\lambda}^{*}$ can be $\mathbf{K}$-embedded in $M$.

iii) $M$ is $\mathbf{K}_{<\lambda}$ chain-universal if each $(<\lambda,<\lambda)$ chain can be $\mathbf{K}$-embedded in $M$.

Applying Theorem 5.6 and Assumption 5.1 ii) $((<\lambda,<\lambda)$-boundedness $)$ it is easy to see:

5.11 Lemma. If $M$ is $\mathbf{K}_{<\lambda}^{*}$-universal then $M$ is $\mathbf{K}_{<\lambda}$ chain-universal.

\subsection{Definition.}

i) $M$ is $\left(\mathbf{K}_{\lambda}, \leq\right)$-homogenous-universal if $M$ is $\mathbf{K}_{<\lambda}$-universal and for each $N_{0} \leq N_{1} \in \mathbf{K}_{<\lambda}$ any $\mathbf{K}$-embedding of $N_{0}$ into $M$ can be extended to a K-embedding of $N_{1}$ into $M$. 
ii) $M$ is strongly $\left(\mathbf{K}_{\lambda}, \leq\right)$-homogenous-universal if $M$ is $\mathbf{K}_{<\lambda}$-universal and each isomorphism between $\mathbf{K}$-substructures of $M$ with power $<\lambda$ can be extended to an automorphism of $M$.

iii) $M$ is chain homogeneous-universal (for $\mathbf{K}_{<\lambda}^{*}$ ) if $M$ is $\mathbf{K}_{<\lambda}^{*}$-universal and for any pair $\underline{M} \prec \underline{N}$ of members of $\mathbf{K}_{<\lambda}^{*}$, any $\mathbf{K}$-embedding of $\underline{M}$ into $M$ can be extended to an embedding of $\underline{N}$ into $M$.

iv) $M$ is strongly chain homogeneous-universal (for $\mathbf{K}_{<\lambda}^{*}$ ) if $M$ is $\mathbf{K}_{<\lambda^{-}}^{*}$ universal and whenever the $(<\lambda,<\lambda)$-chains $\underline{M}, \underline{N} \in \mathbf{K}_{\lambda}^{*}$ are isomorphic and $\mathbf{K}$-embedded in $M$, the isomorphism can be extended to an automorphism of $M$.

We quickly summarise the relations among these notions and then proceed to supporting examples. It is immediate from the definition that a $\left(\mathbf{K}_{\lambda}, \leq\right)$ homogenous-universal model is $\mathbf{K}_{<\lambda^{+}}$-universal. If the empty structure is a $\mathbf{K}$-submodel of every structure the requirement of $\mathbf{K}_{<\lambda}$-universality in the definition of a $\left(\mathbf{K}_{\lambda}, \leq\right)$-homogenous-universal model is redundant. Clearly, Definition 5.12 ii) implies Definition 5.12 i). But in general Definition 5.12 ii) is stronger than Definition $5.12 \mathrm{i}$ ). If we do not require the counterexample to have cardinality $\lambda$ this is true even in the first order case. For there are $\lambda$-saturated structures (thus satisfying i)) that are rigid. (E.g. rigid real closed fields [10].) These notions are however equivalent if $\mathbf{K}$ is smooth (and with the $\lambda$-Löwenheim Skolem property).

Similarly iv) is stronger than iii). We now show notion i) is stronger than notion iii).

5.13 Lemma. If $\mathbf{K}$ is $(<\lambda,<\lambda)$-bounded and $M$ is a $\left(\mathbf{K}_{\lambda}, \leq\right)$-homogenousuniversal model then $M$ is chain homogeneous-universal.

Proof. Let $\underline{M}$ be in $\mathbf{K}_{<\lambda}^{*}$ and $\mathbf{K}$-embedded in $M$. Suppose $\underline{M} \prec \underline{N}$. By the $<\lambda$-Löwenheim Skolem property there is an $M_{1}$ with $\left|M_{1}\right|<\lambda$ and $\underline{M}$ is $\mathbf{K}$-embedded in $M_{1} \leq M$. By the boundedness there exists a model $M_{2}$ that is a $\mathbf{K}$-extension of $\underline{N}$. Since $\underline{M}$ is an amalgamation base, $M_{1}$ and $M_{2}$ can be amalgamated over $\underline{M}$ into some $M_{3}$ (with cardinality $<\lambda$ by the $<\lambda$ Löwenheim Skolem property.) Since $M$ is $\left(\mathbf{K}_{\lambda}, \leq\right)$-homogenous-universal, there is an embedding of $M_{3}$ into $M$ and the image of $\underline{N}$ verifies that $M$ is chain homogeneous-universal. 
5.14 Notation. For some examples the $\lambda$ is not an important parameter. In these cases, we simply omit mentioning any parameter. This means any reasonable choice of $\lambda$ works.

5.15 Example. We define several classes $\mathbf{K}$ such that at each $\lambda$, $\mathbf{K}$ has chain homogeneous-universal models but has no $\mathbf{K}_{<\lambda}$-homogeneous-universal models.

i) Let $\mathbf{K}$ be the class of all structures of the form $L_{U}=\left(L,<, R_{U}\right)$ that are described below with $\leq$ being the usual notion of substructure. $(L,<)$ is a linear order with a last element. For an arbitrary but fixed subset $U$ of $L$, let $R_{U}(x, y)$ hold if $x \in U \& x<y$. $\mathbf{K}$ is the class of structures $\left(L,<, R_{U}\right)$. Each member of $\mathbf{K}$ satisfies the following two sentences.

$$
\begin{gathered}
(\forall x)(\forall y)[R(x, y) \rightarrow x<y] . \\
(\forall x)[(\exists y) R(x, y) \rightarrow(\forall z)[z>x \rightarrow R(x, z)]] .
\end{gathered}
$$

Now the key point is that if $M \in K$ and $x$ is not the last element of $M, x \in U$ is a $\Delta_{1}^{0}$ property of $x$ so $U$ is preserved by extension except that the last element $e$ of $M$ may be in $U$ in some extensions but not others. To see $M$ is not an amalgamation base choose an extension with $e \in U$ and another where it is not.

Now let $\underline{N}=\left\langle N_{i}: i<\omega\right\rangle$ be an increasing chain of members of $\mathbf{K}$ such that $N_{i+1}$ contains an element above all those in $N_{i}$. Then $\bigcup \underline{N} \notin \mathbf{K}$ since it doesn't have a last element. But $\underline{N}$ is in $\mathbf{K}^{*}$ since whether $a \in U$ has been determined for each $a \in \bigcup \underline{N}$.

Thus $\mathbf{K}$ does not have any $\mathbf{K}$-homogenous-universal models (as this implies the amalgamation property for models of smaller size.) But $\mathbf{K}^{*}$ is nonempty and we obtain $\mathbf{K}^{*}$-homogeneous-universal models as in Theorem 5.17. This example has many models in each cardinality.

ii) Let $L$ have a single binary relation $R$. Again let $\leq$ be the usual notion of substructure. Let $T$ assert that $R$ is asymmetric $(x R y \rightarrow \neg y R x)$ and irreflexive, that each point is related to at most one other, and that every point but one, which is related to no one, is related to exactly one other. Then $x R y \vee y R x \vee x=y$ defines an equivalence relation 
such that all classes but one (with a single element) have two elements. Now there is a $\Delta_{1}^{0}$-definition of the predicate " $x$ is the 'lower' of two related elements" (provided $x$ is related to some element). No model in $\mathbf{K}$ is an amalgamation base (the element in the 1-element class can be 'upper' or 'lower' in an extension). $\mathbf{K}$ is $(<\infty,<\infty)$-bounded but is not $(<\lambda,<\lambda)$-closed for any $\lambda$. For any $\lambda$, Player AM has a winning strategy for Game $2(\lambda,<\lambda)$ so by the proof of Theorem 5.17 there are chain homogeneous-universal models but as in the previous example they cannot be $\mathbf{K}_{<\lambda}$-homogeneous-universal. $\mathbf{K}$ is categorical in all infinite powers.

iii) If we vary this example by allowing $\mathbf{K}$ to contain the structures where all classes have two elements then $\mathbf{K}$ is $(\infty, \infty)$ closed but the main point of the example continues to hold. There are now two models in each cardinality.

The last two examples show there is little hope of finding $\mathbf{K}_{\lambda}$-homogeneousuniversal models in the abstract setting solely from hypotheses about the number of models. We show that such models exist and are unique under an amalgamation hypothesis in Theorem 5.25.

5.16 Example. Here is a class $(\mathbf{K}, \leq)$ and a model that is $\mathbf{K}_{<\lambda}$-homogeneousuniversal and chain homogeneous-universal but $\mathbf{K}$ is not smooth. $M \in \mathbf{K}$ if $M$ is isomorphic to a substructure which is closed under subsequence of $\left\langle\lambda^{\leq \omega+1},<, L_{i}\right\rangle$ where $<$ is the natural order by subsequence and the $L_{i}$ are predicates for levels. $M \subseteq N$ is a $\mathbf{K}$-substructure of $N$ if any infinite chain in $M$ that is bounded in $N$ is also bounded in $M$. Now if $M$ has no finite branchs and for each finite initial segment of a branch through $M$ there are $\lambda$ branches with an upper bound and $\lambda$ branches with no upper bound, $M$ is $\mathbf{K}_{<\lambda}$-homogeneous-universal and so chain homogeneous universal. But any model (or chain of models) that contains an infinite branch without an upper bound is not an amalgamation base.

For the remainder of this section we explore the properties of notions iii) and iv). We first prove the existence of a $\mathbf{K}_{<\lambda}^{*}$ chain homogeneousuniversal model. Then we will show using the assumption that $\mathbf{K}$ has few models any such $\mathbf{K}_{<\lambda}^{*}$ chain homogenous-universal model is strongly $\mathbf{K}_{<\lambda}^{*}$ chain homogeneous and thus there is a unique $\mathbf{K}_{<\lambda}^{*}$ chain homogeneousuniversal model of power $\lambda$. 
5.17 Theorem. Suppose $\lambda$ satisfies the assumptions enumerated in Section 1 and that Player $B$ has a winning strategy for Game $1(\lambda, \lambda)$. There is $a \mathbf{K}_{<\lambda}^{*}$ chain homogeneous-universal model with cardinality $\lambda$.

Proof. We construct a sequence of models $\left\langle M_{i}: i<\lambda\right\rangle$ of cardinality $<\lambda$ with the universe of $M_{i}$ contained in $\lambda$; the required $M$ is $\bigcup M_{i}$. Since $\lambda^{<\lambda}=\lambda\left(\right.$ as $\nabla_{\lambda, \kappa, R}(S)$ holds), we can let $\left\langle\underline{N}_{i}: i<\lambda\right\rangle$ enumerate a set of representatives (say with universe contained in $\lambda$ ) of all isomorphism types of members of $\mathbf{K}_{<\lambda}^{*}$.

Having constructed $M_{i}$ we define $M_{i+1}$ with cardinality $<\lambda$ by a play of Game $1(<\lambda, \lambda)$. We construct a sequence $\left\langle M_{i, j}: j<\alpha\right\rangle$ of structures with universe contained in $\lambda$ where $\alpha=|i|+\left|M_{i}\right|^{\left|M_{i}\right|}<\lambda$. The $M_{i, j}$ will be the plays of Player B in the game. The plays of his opponent, denoted $M_{i, j}^{\prime}$ will guarantee the homogeneity. Let $G_{j}=\left\langle\underline{A}_{j}, \underline{B}_{j}, f_{j}\right\rangle$ for $j<\alpha$ be a list of all triples such that $\underline{A}_{j}, \underline{B}_{j} \in \mathbf{K}_{<\lambda}^{*}, \cup \underline{A} \subseteq \lambda, \cup \underline{B} \subseteq \lambda, \underline{A}_{j} \prec \underline{B}_{j}, \underline{B}_{j} \approx \underline{N}_{\beta}$ for some $\beta<i$, and $f_{j}$ maps $\underline{A}_{j}$ into $M_{i}$. Now $M_{i}, 0=M_{i}$ and $M_{i, \delta}^{\prime}=M_{i, \delta}$ for $\delta$ a limit ordinal. $M_{i, j+1}^{\prime}$ is an amalgam of $M_{i, j}$ with $\underline{B}_{j}$ over $\underline{A}_{j}$ with the universe of $M_{i, j+1}$ contained in $\lambda . M_{i, j}$ is an extension of $M_{i, j}^{\prime}$ with universe a proper subset of $\lambda$ chosen by Player B's winning strategy. If $\delta$ is a limit ordinal $\leq \alpha, M_{i, \delta}$ is chosen as a bound for $\left\langle M_{i, j}: j<\delta\right\rangle$ with cardinality $<\lambda$ by Player B's winning strategy. $M_{i, \delta}$ of the proper cardinality can be chosen by the regularity of $\lambda$ and the $<\lambda$-Löwenheim Skolem property. Let $M_{i+1}^{\prime}$ be $M_{i, \alpha}$ and to guarantee universality let $M_{i+1}$ be a common $\mathbf{K}$-extension of $M_{i+1}^{\prime}$ and $\underline{N}_{i+1}$.

The last result may be vacuous (e.g. nothing in the hypotheses guarantees that $\mathbf{K}_{<\lambda}^{*}$ is not empty.) Adding Assumption 5.1 and applying Lemma 5.11 we deduce:

5.18 Corollary. Suppose $\lambda$ and $\mathbf{K}$ satisfy the assumptions enumerated in Paragraphs 1.1, 5.1, 5.9 and $\lambda^{<\lambda}=\lambda$. There is a $\mathbf{K}_{<\lambda}^{*}$ chain homogeneousuniversal model with cardinality $\lambda$ that is $\mathbf{K}_{\lambda}$ universal.

We will now show that if $\mathbf{K}$ has few models of power $\lambda$ then any chain homogeneous-universal model is actually strongly chain homogeneous-universal and thus the uniqueness of the chain homogeneous-universal model. The keys to showing this lemma are the following invariants.

Expand the language $L$ of our class $K$ to $L^{*}$ by adding for each $\alpha<\lambda$ an $\alpha$-ary predicate $P_{\alpha}$. Expand each $L$-structure $M$ to an $L^{*}$-structure $M^{*}$ by 
letting $P_{\alpha}$ hold of an $\alpha$-sequence $\bar{a}$ just if $\bar{a}$ enumerates a $\mathbf{K}$-submodel of $M$. Without loss of generality the universe of $M$ is $\lambda$. As in [9], for each $\beta<\lambda$, $\phi_{\beta^{*}}$ describes the $\infty \lambda$-type of $\beta$ (i.e. of $\langle\gamma: \gamma<\beta\rangle$ ). We denote by $\bar{x}_{j}$ the sequence of variables $\left\langle x_{i}: i<j\right\rangle$.

5.19 Definition. For any representation $\underline{M}$ of a model $M$,

$S(M, \underline{M})=\left\{i: i<\lambda\right.$ is a limit ordinal and $\left.\left.M \models \neg\left(\forall \bar{x}_{i}\right) \bigwedge_{j<i} \phi_{j^{*}}\left(\bar{x}_{j}\right) \rightarrow \phi_{i^{*}}\left(\bar{x}_{i}\right)\right)\right\}$.

$\hat{S}(M, \underline{M})=\left\{i: i<\lambda\right.$ is a limit ordinal and $\left.\left\langle M_{j}: j<i\right\rangle \notin \mathbf{K}_{<\lambda}^{*}\right\}$.

It is easy to see that $S(M, \underline{M})$ is an invariant of $M$ modulo the cub filter; thus we abbreviate $S(M, \underline{M})$ to $S_{M}$. The same holds for $\hat{S}$. Thus, $S_{M}$ is exactly the invariant defined in [9]. (It appears to be the complement but this is a typo in the earlier paper.) For $X \subset \lambda$, we write $X^{c}$ for $\lambda-X$.

5.20 Claim. If $M$ is chain homogeneous-universal $S_{M}$ and $\hat{S}_{M}$ are equivalent modulo the cub filter on $\lambda$.

Proof. We say that an $L_{\infty, \lambda}$ formula $\phi(\bar{x})$ is complete if it implies the quantifier free diagram of $\bar{x}$; denote this formula by $\phi^{*}(\bar{x})$. Now we will show that for each complete formula $\phi(\bar{x}) \in L_{\infty, \lambda}$ such that $\phi(\bar{x})$ implies that $\bar{x}$ enumerates an $\mathbf{K}$-increasing chain, and for any sequence $\bar{a} \in M$ that enumerates a member of $\mathbf{K}_{<\lambda}^{*}$,

$$
M \models \phi(\bar{a}) \text { if and only if } M \models \phi^{*}(\bar{a}) .
$$

We prove this assertion by induction on quantifier rank; for quantifier free formulas it is tautologous. Suppose $\phi(\bar{x})$ is $(\exists \bar{v}) \theta(\bar{v}, \bar{x})$ and by induction that we have the proposition for $\theta$. Fix any $\bar{b} \in M$ that enumerates a member of $\mathbf{K}_{<\lambda}^{*}$ and $M \models \phi^{*}(\bar{b})$. We must show $M \models \phi(\bar{b})$. Suppose $\phi(\bar{a})$ is witnessed by $\theta\left(\bar{a}_{1}, \bar{a}\right)$. By Lemma 5.6 there is an $\bar{a}_{2} \subseteq M$ such that $\bar{a}_{1} \bar{a}_{2} \bar{a}$ enumerates a member of $\mathbf{K}_{<\lambda}^{*}$. Let $\delta(\bar{v}, \bar{w}, \bar{x})$ be the conjunction of the quantifier-free diagram of $\bar{a}_{1} \bar{a}_{2} \bar{a}$ and $\phi^{\prime}(\bar{v}, \bar{w}, \bar{x})$ denote $\theta(\bar{v}, \bar{x}) \wedge \delta(\bar{v}, \bar{w}, \bar{x})$. Since $M$ is chain homogeneous-universal, there exist $\bar{b}_{1}, \bar{b}_{2} \subseteq M$ such that $\bar{b}_{1} \bar{b}_{2} \bar{b}$ enumerates a member of $\mathbf{K}_{<\lambda}^{*}$ isomorphic to $\bar{a}_{1} \bar{a}_{2} \bar{a}$. By induction, we have $\models \phi^{\prime}\left(\bar{b}_{1}, \bar{b}_{2}, \bar{b}\right)$ and thus $\models \phi(\bar{b})$ as required.

Now suppose $i \in \hat{S}_{M}^{c}$. Then $M \mid i$ is an amalgamation base and we have just observed that the $\infty \lambda$ type of $i$ is equivalent to a quantifier free formula. So $i$ is certainly not in $S_{M}$. Thus $\hat{S}_{M}^{c} \subseteq S_{M}^{c}$. 
The set $C=\left\{\delta<\lambda: \delta=\sup \left(\delta \cap \hat{S}_{M}^{c}\right) \& \delta\right.$ is the universe of $\left.M \mid \delta\right\}$ is a cub. To complete the proof, it suffices to show $\hat{S}_{M} \cap C \subseteq S_{M}$. (This yields $\hat{S}_{M}=S_{M}$ modulo the cub filter.) But if $i \in \hat{S}_{M}, \underline{M} \mid i \notin \mathbf{K}_{<\lambda}^{*}$. So there exist $(<\lambda,<\lambda)$ chains $\underline{M}^{\prime}$ and $\underline{M}^{\prime \prime}$ whose restriction to $i$ are isomorphic to $\underline{M}$ but which are incompatible over $\underline{M} \mid i$. Since $M$ is $<\lambda$ chain-universal there are copies of both $\underline{M}^{\prime}$ and $\underline{M}^{\prime \prime} \mathrm{K}$-embedded in $M$. Note that if $i \in C$, the $\infty \lambda$ type of $i$ is equivalent to a quantifier free formula. Thus $\underline{M}^{\prime} \mid i$ and $\underline{M}^{\prime \prime} \mid i$ have the same $\infty \lambda$-type and so witness that $i \in S_{M}$.

5.21 Assumption. We assume for the next theorem the combination of $\square$ and $\diamond$ described on page 7 of $[9]$.

5.22 Theorem. If there is an $M \in \mathbf{K}$ such that $\hat{S}_{M}$ is stationary then there are $2^{\lambda}$ models of $\mathbf{K}$ with power $\lambda$.

Proof. Since $\hat{S}_{M}=S_{M}$ we can just quote the main result of [9]. It is shown in that paper, that for $\lambda$ satisfying the set theoretic principle described there, each model $M$ of power $\lambda$ is categorical in the language $L_{\infty, \lambda}$ if $S_{M}$ is not stationary or has $2^{\lambda}$ models of power $\lambda$ that are $L_{\infty, \lambda}$-equivalent to it if $S_{M}$ is stationary. The $2^{\lambda}$ models defined in the proof are all unions of members of $\mathbf{K}_{<\lambda}$ so since $\mathbf{K}$ is $(<\lambda, \lambda)$-closed we have the result.

5.23 Theorem. Suppose $M$ has cardinality $\lambda, \hat{S}_{M}$ is not stationary, $M$ is chain homogeneous-universal then $M$ is strongly chain homogeneous-universal. More generally, if $M$ and $N$ are any two chain homogenous-universal models and neither $\hat{S}_{M}$ nor $\hat{S}_{N}$ is stationary then $M$ and $N$ are isomorphic.

Proof. This is the other half of the dichotomy proved in [9]. The argument is presented in detail there. To summarise, suppose $M$ and $N$ are each $\mathbf{K}_{<\lambda}^{*}$ chain homogeneous-universal. Construct by induction a back and forth between $M$ and $N$. The successor stages are easy by the definition of $\mathbf{K}_{<\lambda^{-}}^{*}$ homogeneous. The tricky point is the limit stage. But since $\hat{S}_{M}$ and $\hat{S}_{N}$ are not stationary we can (by restricting to a cub) assume they are empty. Thus at limit stages the sequences already constructed in $M$ and $N$ are in $\mathbf{K}_{<\lambda}^{*}$. So we can apply the definition of $\mathbf{K}_{<\lambda}^{*}$-homogeneity and continue the construction. 
5.24 Corollary. Suppose $\lambda$ and $\mathbf{K}$ satisfy the assumptions enumerated in Paragraphs 1.1, 5.1, 5.9. There is a unique $\mathbf{K}_{<\lambda}^{*}$ chain homogeneous-universal model with cardinality $\lambda$ and it is $\mathbf{K}_{<\lambda}^{*}$ strongly chain homogeneous-universal.

5.25 Theorem. Suppose for each $\kappa<\lambda$ and some $R \leq \lambda$, $\mathbf{K}$ and $\lambda$ satisfy the assumptions enumerated in Paragraphs 1.1 and 5.1. Suppose further that $\mathbf{K}$ satisfies the amalgamation and joint embedding propeties and $\lambda^{<\lambda}=\lambda$. There is a unique $\left(\mathbf{K}_{\lambda}, \leq\right)$ homogeneous-universal model with cardinality $\lambda$

Proof. With amalgamation it is straightforward to construct an $\left(\mathbf{K}_{<\lambda}, \leq\right)$ homogeneous-universal model. It is chain homogeneous-universal and thus unique.

While we assumed $\bigotimes_{\lambda, \kappa, R}$ for each $\kappa<\lambda$, this is excessive. We only need this assumption for those $\kappa$ where there is a possible failure of smoothness. The following result does not use the hypothesis that $\mathbf{K}$ has few models.

5.26 Theorem. The following are equivalent.

i) $S_{M}$ is not stationary for some $\mathbf{K}_{<\lambda}^{*}$ chain homogeneous-universal model $M$ of power $\lambda$;

ii) Player AM has a winning strategy in Game $2(\lambda,<\lambda)$.

Proof. First we show i) implies ii). Let $\underline{M}$ be a representation of $\underline{M}$ such that $S_{M}$ is not stationary; fix a closed unbounded set $C$ that is disjoint from $S_{M}$. To win player AM chooses $P_{i}$ as a $\mathbf{K}$-extension of $L_{i}$ and an isomorphism $f_{i}$ (extending the $f_{j}$ for $j<i$ ) of $P_{i}$ with an $M_{j_{i}}$ whose universe is an ordinal in $C$. Thus, each limit stage in the chain constructed by the play of the game is in $C$ and so is an amalgamation base as required.

To see ii) implies i) modify the proof of Theorem 5.17. Construct a sequence $M_{i, j}^{\prime}$ as follows. Play Game $2(\lambda,<\lambda)$. Let player NAM's moves be $L_{j}=M_{i, j}$ where $M_{i, j}$ is chosen just as in the proof of Theorem 5.17. Let $P_{j}=M_{i, j}^{\prime}$ be chosen according to Player AM's winning strategy. This guarantees that letting $M^{\prime}=\bigcup_{i, j} M_{i, j}^{\prime}, S\left(M^{\prime}, \underline{M^{\prime}}\right)$ is not stationary (indeed empty).

5.27 Extension. If $\lambda=\kappa^{+}$and $\square_{\lambda}$ holds ( $\square_{\kappa}$ in Jensen's notation) then Condition ii) in the last lemma can be replaced by Game $2(\lambda, \kappa)$. 


\section{Conclusions and Problems}

Much of this paper can be viewed as answering the question: What is the role of closure under union in the construction of homogeneous-universal models? We have the following symbolic equation:

closure under unions $=$ boundedness + smoothness.

We have shown that the boundedness hypothesis can be weakened to the existence of a winning strategy for Player B in Game 1; this suffices to show the existence of $\mathbf{K}_{\lambda}^{*}$ chain homogeneous-universal models. Similarly, we have weakened smoothness to the nonexistence of a winning strategy for Player NAM in game 2; this suffices to prove the chain homogeneous-universal is $\mathbf{K}_{<\lambda}$ chain-universal. We are then able to apply the argument from [9] to show that if $\mathbf{K}$ has few models the chain homogeneous-universal model is unique. The most obvious question is

1 Question. Can the results of this paper be obtained in $Z F C$ ?

Rami Grossberg has made progress on this question and several related ones. In particular he has shown that there is a model of $\mathrm{ZFC}+\mathrm{CH}+$ $2^{\aleph_{1}}=2^{\aleph_{2}}$ in which there is a class $\mathbf{K}$ that satisfies the model theoretic conditions of Theorem 4.1 (with $\lambda=\aleph_{2}$ ) but has only one model of power $\aleph_{2}$. In the case where $\lambda$ is a successor cardinal Grossberg has weakened our set theoretic assumptions. These results are still being written up.

But there is another use of closure under unions. Separating one of the components of the notions of limit model defined in [7, 12] and generalizing [2], call a structure $M \mathbf{K}$-rich if it is $\mathbf{K}$-isomorphic to a proper substructure. Now it is easy to see that if a structure of power $\lambda$ is rich and $\mathbf{K}_{\leq \lambda}$ universal and if $\mathbf{K}$ is closed under union then there is a member of $\mathbf{K}$ with power $\lambda^{+}$. There are examples [12, page 431] of classes $\mathbf{K}$ that are $\lambda$-categorical such that the model of power $\lambda$ is universal, maximal, and homogenous (since rigid).

2 Question. Suppose $\mathbf{K}$ has few models in power $\lambda$. What are minimal model theoretic conditions on a class $\mathbf{K}$ so that the homogeneous-universal model is not maximal? Can the homogeneous-universal model be a Jonsson model? 
3 Question. What sort of transfer theorems can be proved for the existence of $\mathbf{K}_{\lambda}^{*}$-homogeneous-universal models (in various $\lambda$ ).

Much of our efforts have been dedicated to showing the uniqueness of the homogeneous-universal model. This raises a metatheoretical question.

4 Question. How much of stability theory can be carried through in abstract class $\mathbf{K}$ that has homogeneous-universal models (in many cardinalities) but where they may not be unique.

\section{Appendix: Set Theoretic Lemmas}

In this section we include the proofs of the two combinatorial arguments used in the main arguments and some comments on how they can be extended.

7.1 Lemma. [Proof of Lemma 3.4] Suppose $\mu^{\kappa}=\mu$ and $2^{\mu}=\mu^{+}=\lambda$. If $S^{*} \subseteq C^{\kappa}(\lambda)$ is stationary then there is an $S \subseteq \lambda$ such that $\otimes_{\lambda, \kappa, \omega}$ holds and $S \cap C^{\kappa}(\lambda) \subseteq S^{*}$.

Proof. Let $S^{*} \subseteq C^{\kappa}(\lambda)$ be stationary. Let $\left\langle A_{\alpha}: \alpha<\lambda\right\rangle$ be a list of all bounded subsets (indeed $A_{\alpha} \subseteq \alpha$ ) of $\lambda$ each appearing $\lambda$ times. (Such a list exists since $2^{\mu}=\mu^{+}=\lambda$.) Let $\left\{\left\langle A^{\alpha, \epsilon}, C^{\alpha, \epsilon}\right\rangle: \epsilon<\mu\right\}$ list all pairs $\langle A, C\rangle$ where $A$ has the form $\cup_{i \in X} A_{i}$ with $X$ a subset of $\alpha$ of cardinality at most $\kappa$ and $C$ is a closed subset of $\alpha$ with $\operatorname{otp}(C) \leq \kappa$. Moreover, we require that if $\operatorname{otp}(C)=\kappa$ then $\alpha \in S^{*}$. There are only $\mu$ such pairs since $\mu^{\kappa}=\mu$.

By Engelking and Karlowicz [1], there exists a sequence of functions $F_{\xi}$ : $\lambda \mapsto \mu$ for $\xi<\mu$ such that any partial function $h$ taking $\lambda$ to $\mu$ with $|\operatorname{dom} h| \leq$ $\kappa$ extends to some $F_{\xi}$.

Let $\langle\rangle,, \operatorname{pr}_{0}, \operatorname{pr}_{1}$, denote pairing and projection functions on $\mu$ and on $\lambda$ such that $\alpha$ and $\beta$ are always less than or equal to $\langle\alpha, \beta\rangle$.

For each $\zeta<\mu$, we will define two sequences of sets $\mathcal{A}^{\zeta}=\left\langle A_{\alpha}^{\zeta}: \alpha<\lambda\right\rangle$ and $\mathcal{C}^{\zeta}=\left\langle C_{\alpha}^{\zeta}: \alpha<\lambda\right\rangle$ and a set $S^{\zeta}$. We will show that for some $\zeta, \mathcal{A}_{\zeta}$ and $\mathcal{C}^{\zeta}$ satisfy the definition of $\otimes_{\lambda, \kappa, \omega}\left(S^{\zeta}\right)$.

For any function $F$, let $F^{0}$ and $F^{1}$ denote the result of applying the first and second projection functions respectively after $F$.

If $C^{\alpha, F_{\zeta}^{0}(\alpha)}$ is closed and for each $\beta \in C^{\alpha, F_{\zeta}^{0}(\alpha)}, C_{\beta}^{\zeta}=\beta \cap C^{\alpha, F_{\zeta}^{0}(\alpha)}$ and, if $\alpha$ is a limit ordinal, $\alpha=\sup C^{\alpha, F_{\zeta}^{0}(\alpha)}$ while each nonaccumulation point 
of $C^{\alpha, F_{\zeta}^{0}(\alpha)}$ is an even successor ordinal, let $C_{\alpha}^{\zeta}=C^{\alpha, F_{\zeta}^{0}(\alpha)}$. Otherwise, let $C_{\alpha}^{\zeta}=\emptyset$.

As a first approximation to $A_{\alpha}^{\zeta}$, let $B_{\alpha}^{\zeta}=\left\{\gamma\left\langle\alpha:\langle\zeta, \gamma\rangle \in A^{\alpha, F_{\zeta}^{1}(\alpha)}\right\}\right.$. Now define $A_{\alpha}^{\zeta}$ by induction on $\alpha$. At stage $\alpha$, if for each $\beta \in \operatorname{acc}\left[C_{\alpha}^{\zeta}\right], A_{\beta}^{\zeta}=B_{\beta}^{\zeta} \cap \beta$, then $A_{\alpha}^{\zeta}=B_{\alpha}^{\zeta}$; otherwise $A_{\alpha}^{\zeta}=\cup_{\beta \in \operatorname{acc}\left[C_{\alpha}^{\zeta}\right]} A_{\beta}^{\zeta}$.

Let $S_{1}^{\zeta}=\left\{\delta \in S^{*}: \delta=\sup C_{\delta}^{\zeta}\right\}$. Finally, let $S^{\zeta}=S_{1}^{\zeta} \cup \cup_{\delta \in S_{1}^{\zeta}} C_{\delta}^{\zeta}$.

Now we claim that for some $\zeta, \mathcal{A}^{\zeta}$ and $\mathcal{C}^{\zeta}$ satisfy the definition of ${ }_{\lambda, \kappa, \omega}\left(S^{\zeta}\right)$. It is easy to check that for each $\zeta, \mathcal{A}^{\zeta}, S^{\zeta}$ and $\mathcal{C}^{\zeta}$ satisfy all the conditions except possibly ii) and vii). (This would be possible even if all the $C_{\alpha}^{\zeta}$ were empty.)

Moreover, $A_{\alpha}^{\zeta} \subseteq \alpha$ and if $\beta \in \operatorname{acc}\left[C_{\alpha}^{\zeta}\right], A_{\beta}^{\zeta}=A_{\alpha}^{\zeta} \cap \beta$. So the nontrivial point is to check vii) b) of 3.2. If for some $\zeta \mathcal{A}^{\zeta}, S^{\zeta}$, and $\mathcal{C}^{\zeta}$ work, we are finished; if not for each $\zeta$ there is a counterexample $\left\langle A_{\zeta}^{*}, E_{\zeta}\right\rangle$. That is, there is some cub $D_{\zeta}$ with $D_{\zeta} \cap X_{E_{\zeta}}=\emptyset$. Interpreting the definition of $X_{E}$ (from vii) of Definition 3.2), $X_{E_{\zeta}}=\left\{\delta \in S_{1}^{\zeta}: \delta \in E_{\zeta} \& \operatorname{acc}\left[C_{\delta}\right] \subseteq E_{\zeta} \& A_{\delta}^{\zeta}=A_{\zeta}^{*} \cap \delta\right\}$. Since $S_{1}^{\zeta} \subseteq X_{E_{\zeta}}$ showing $X_{E_{\zeta}}$ stationary also satisfies condition ii). Let $A^{*}=\left\{\langle\zeta, \alpha\rangle: \zeta<\mu \& \alpha \in A_{\zeta}^{*}\right\}$. (Note that by the definition of $\langle$,$\rangle , this is$ a set of ordinals). Let $D=\cap_{\zeta<\mu} D_{\zeta}$.

Let $E$ be the set of $\delta \in \cap_{\zeta<\mu} E_{\zeta}$ such that $\delta$ is closed under the pairing and projection functions and for each $\alpha<\delta, A^{*} \cap \alpha \in\left\{A_{i}: i<\delta\right\}$.

Define a function $g: \lambda \mapsto \lambda$ by $g(i)$ is the least $j$ such that $A^{*} \cap i=A_{j}$. Since the $A_{i}$ enumerate all bounded subsets of $\lambda$ this function is well defined. Note that $i<\alpha$ is in $E$ implies $g(i)<\alpha$.

Then $E$ is a cub in $\lambda$. To demonstrate a contradiction, fix $\delta \in S^{*} \cap$ $C^{\kappa}(\lambda) \cap \operatorname{acc}[E] \cap D\left(\delta\right.$ exists as $S^{*} \subseteq C^{\kappa}(\lambda)$ is stationary) and then choose a subset $C_{\delta}$ of $\delta$ such that acc $\left[C_{\delta}\right] \subseteq E$, each nonaccumulation point of $C_{\delta}$ is an even successor ordinal, and $\delta=\sup C_{\delta}$.

Let $Y_{\delta}$ denote $\operatorname{acc}\left[C_{\delta}\right] \cup\{\delta\}$.

For each $\alpha \in Y_{\delta}, X_{\alpha}=\left\{g(i): i \in C_{\delta} \cap \alpha\right\}$ is a subset of $\alpha$ with cardinality less than $\kappa$ and so for some $\nu, A^{\alpha, \nu}=\cup_{j \in X_{\alpha}} A_{j}$. But,

$$
\cup_{j \in X_{\alpha}} A_{j}=\cup_{i \in C_{\delta} \cap \alpha} C_{g(i)}=\cup_{i \in C_{\delta} \cap \alpha}\left(A^{*} \cap i\right)=\left(A^{*} \cap \alpha\right)
$$

(as $\alpha \in Y_{\delta}$ ); so $A^{*} \cap \alpha=A^{\alpha, \nu}$. By the choice of $\left\langle C^{\alpha, \epsilon}: \epsilon<\mu\right\rangle$, for some $\nu^{\prime}$, $C_{\delta} \cap \alpha=C^{\alpha, \nu^{\prime}}$.

Based on these two observations we can now define a function $h$ with domain $\{\delta\} \cup C_{\delta}$ as follows. For each $\gamma \in Y_{\delta}$, let $h(\gamma)$ be the minimal $\epsilon<\mu$ 
with $A^{*} \cap \gamma=A^{\gamma, \operatorname{pr}_{1}(\epsilon)}$ and $C_{\delta} \cap \gamma=C^{\gamma, \operatorname{pr}_{0}(\epsilon)}$. For $\gamma \in \operatorname{nacc}\left[C_{\delta}\right]$, we only care about $h_{0}(\gamma)$; let $h(\gamma)$ be the minimal $\epsilon<\mu$ with $C_{\delta} \cap \gamma=C^{\gamma, \operatorname{pr}_{0}(\epsilon)}$.

Now for some $\zeta$, the choice of the $F_{\zeta}$ guarantees that $h \subseteq F_{\zeta}$. For this $\zeta$, we show by induction on $\alpha \in C_{\delta} \cup\{\delta\}$ that $C_{\alpha}^{\zeta}=C^{\alpha, h^{0}}(\alpha)$ (and thus $\delta \in S_{1}^{\zeta}$ ) and $A_{\delta}^{\zeta}=A_{\zeta}^{*} \cap \delta$ (so $\delta \in X_{E_{\zeta}}$ ). This contradicts the choice of $D_{\zeta}$ and completes the proof.

We first show $C_{\alpha}^{\zeta}=C^{\alpha, h^{0}(\alpha)}$ by induction on $\alpha \in C_{\delta} \cup\{\delta\}$. For each such $\alpha, C_{\delta} \cap \alpha=C^{\alpha, h^{0}(\alpha)}$. If $\alpha$ is least in $C_{\delta}$, this implies $C_{\alpha}^{\zeta}=C^{\alpha, h^{0}(\alpha)}$. Suppose the result holds for $\beta \in C_{\delta}$ that are less than $\alpha$. Then by induction for $\beta<\alpha$,

$$
C_{\beta}^{\zeta}=C^{\beta, h^{0}(\beta)}=C_{\delta} \cap \beta=\left(C_{\delta} \cap \alpha\right) \cap \beta=C^{\alpha, h^{0}(\alpha)} \cap \beta .
$$

The requirements of the definition of $C_{\alpha}^{\zeta}$ are met so $C_{\alpha}^{\zeta}=C^{\alpha, h^{0}(\alpha)}$ as required.

A similar induction shows $A_{\alpha}^{\zeta}=A_{\zeta}^{*} \cap \alpha$. Suppose first that $\alpha$ is minimal in $C_{\delta}$. Then by definition, $A_{\alpha}^{\zeta}=\left\{\gamma:\langle\zeta, \gamma\rangle \in A^{\alpha, F_{\zeta}^{1}(\alpha)}\right\}$. But $h \subseteq F_{\zeta}$ so $A^{*} \cap \alpha=A^{\alpha, F_{\zeta}^{1}(\alpha)}$. Therefore,

$$
A_{\alpha}^{\zeta}=B_{\alpha}^{\zeta}=\left\{\gamma:\langle\zeta, \gamma\rangle \in A^{*} \cap \alpha\right\}=A_{\zeta}^{*} \cap \alpha .
$$

Now suppose that for $\beta \in C_{\delta}$ with $\beta<\alpha$,

$$
A_{\beta}^{\zeta}=A_{\zeta}^{*} \cap \beta=B_{\beta}^{\zeta} .
$$

To see this equality holds for $\alpha$ note that by definition of $A_{\alpha}^{\zeta}$, this yields $A_{\alpha}^{\zeta}=B_{\alpha}^{\zeta}$. But $B_{\alpha}^{\zeta}=A_{\zeta}^{*} \cap \alpha$ since $h \subseteq F_{\zeta}$.

We have established Lemma 3.4 in ZFC. The specific requirements on $\kappa$ and $\lambda$ can be weakened if $V=L$. We would like to remove the restriction that $R=\omega$ and that $\mu^{\kappa}=\mu$. The principal $\mathbf{S D}_{\lambda}$ is established for all uncountable successor cardinals $\lambda=\mu^{+}$in [3] assuming $V=L$. This principal differs from $\nabla_{\lambda, \kappa, R}(S)$ in three ways: the cubs $C_{\alpha}$ are defined only for limit ordinals $\alpha$, the cardinal $\kappa$ is identified with $\mu$, there is no explicit treatment of $R$. In fact, $\mathbf{S D}_{\lambda}$ describes the default case where $R=\lambda$. Since we assume $\mu^{\kappa}=\mu$, our proof of Lemma 3.4 definitely misses the case $\kappa=\mu$.

We outline technical modifications of a system $\mathcal{C}$ satisfying $\mathbf{S D}_{\lambda}$ to show

Lemma $7.2(\mathbf{V}=\mathbf{L})$. [Proof of Lemma 3.5] If $\lambda=\mu^{+}$and $\kappa \leq \mu$, then for some stationary $S, \otimes_{\lambda, \kappa, \lambda}(S)$. 
Proof. We show how to use to $C_{\alpha}$ from $\mathbf{S D}_{\lambda}$ to construct first a sequence $\mathcal{C}^{1}$ such that ${ }_{\lambda, \mu, \lambda}(S)$ holds with $S=\lambda$. Let $E$ be the set of ordinals $>0$ in $\lambda$ divisible by $\mu$ and let $E^{*}$ denote the accumulations points of $E$.

We define by induction on $\alpha \in E$ a sequence, increasing with $\alpha, \underline{C}^{1} \mid(\alpha+$ 1) $=\left\langle C_{\beta}^{1}: \beta \leq \alpha\right\rangle$.

i) $\alpha=\min E$ : Thus, $\alpha=\mu$. If $\beta$ is even $C_{\beta}^{1}$ is the set of even ordinals less than $\beta$; if $\beta$ is odd, $C_{\beta}^{1}$ is empty.

ii) $\alpha$ is a successor in $E$ : Thus $\alpha=\alpha^{*}+\chi$ where $\alpha^{*} \in E$ and $\underline{C}^{1} \mid\left(\alpha^{*}+1\right)$ has been defined. Fix a map $h_{\alpha}$ from $\mu$ onto $\alpha^{*}$.

(a) $\alpha^{*}<\beta \leq \alpha$ and $4 \mid \beta$ : $C_{\beta}^{1}$ consists of those ordinals in $\left(\alpha^{*}, \beta\right)$ that are divisible by 4 .

(b) $\alpha^{*}<\beta \leq \alpha$ and $\beta$ is odd: $C_{\beta}^{1}$ is empty.

(c) $\alpha^{*}<\beta \leq \alpha$ and $4 \not \beta \beta$ but $2 \mid \beta$ : Thus $\beta$ has the form $\alpha^{*}+4 i+2$ for some $i$.

- otp $C_{h(i)}^{1}<\mu$ and $h(i)$ is even: $C_{\beta}^{1}=C_{h_{1}(i)}^{1} \cup\{h(i)\}$

- otherwise: $C_{\beta}^{1}$ is empty.

iii) $\alpha=\delta \in E^{*}$, i.e a limit in $E$ : we only have to define $C_{\delta}^{1}$.

(a) $\operatorname{acc}\left[C_{\delta}\right] \cap E^{*}=\emptyset$ : Necessarily $\operatorname{cf}(\delta)=\aleph_{0}$. Choose an increasing sequence $\left\{\alpha_{n}: n<\omega\right\}$ from $E$ with limit $\alpha$. Then choose by induction on $n$ successor even ordinals $\beta_{n}$ with $\alpha_{n}<\beta_{n}<\alpha_{n}+\mu$ such that $C_{\beta_{m}}^{1}=\left\{\beta_{n}: n<m\right\}$. (The third clause of the previous case is the key to this induction.) Finally, let $C_{\delta}^{1}$ be the set of $\beta_{n}$.

(b) acc $\left[C_{\delta}\right] \cap E^{*} \neq \emptyset$ but acc $\left[C_{\delta}\right] \cap E^{*}$ is bounded in $\delta$ by some $\delta^{\prime}$. Choose $\alpha_{n}$ with limit $\delta^{\prime}$ and $\beta_{n}$ as before but so that $C_{\beta_{n}}^{1}=C_{\delta^{\prime}} \cup$ $\left\{\delta^{\prime}\right\} \cup\left\{\beta_{m}: m<n\right\}$. Then let $C_{\delta}^{1}=C_{\delta^{\prime}} \cup\left\{\delta^{\prime}\right\} \cup\left\{\beta_{m}: m<\omega\right\}$.

(c) $\delta=\sup \left(\operatorname{acc}\left[C_{\delta}\right] \cap E^{*}\right)$ : Let $C_{\delta}^{1}=\cup_{\alpha \in C_{\delta} \cap E} C_{\alpha}^{1}$.

Now one shows by induction on $\alpha$ that if $\beta \in C_{\alpha}^{1}$, then $C_{\alpha}^{1} \cap \beta=C_{\beta}^{1}$. (The other requirements on the $C_{\alpha}^{1}$ are easily verified.)

If $\kappa<\mu$ we modify the $C_{\alpha}^{1}$ defined in the first stage of the proof as follows. If otp $C_{\alpha}^{1} \leq \kappa$ then $C_{\alpha}^{2}=C_{\alpha}^{1}$. If otp $C_{\alpha}^{1}>\kappa$ then $C_{\alpha}^{2}=\left\{\beta \in C_{\alpha}^{1}\right.$ : otp $\left.C_{\beta}^{1}>\kappa\right\}$. Let $S_{1}=\left\{\alpha<\lambda: \operatorname{cf}(\alpha)=\kappa \& \operatorname{otp} C_{\alpha}^{1}=\kappa\right\}$. And $\tilde{S}_{1}=\left\{\alpha<\lambda: \operatorname{otp} C_{\alpha}^{1} \leq\right.$ $\kappa\}$. 


\subsection{Concluding Remarks.}

i) Examination of our proof of Lemma 3.4 shows that in fact given a square sequence, it is possible to add on a diamond sequence to satisfy $\nabla_{\lambda, \kappa, R}(S)$. We state this explicitly in Lemma 7.4 below.

ii) The proof of Lemma 3.5 can be extended to allow for $R<\lambda$.

iii) In fact, the derivation of $\nabla_{\lambda, \kappa, R}$ for $R \leq \lambda$ from the assumption that $\mu=\mu^{\kappa}, \lambda=2^{\mu}$ and there is a square on $\{\delta<\lambda: \operatorname{cf}(\delta)<R\}$ but without assuming $\mathrm{V}=\mathrm{L}$ will be published elsewhere by the second author.

iv) Similar results hold assuming $\mathrm{V}=\mathrm{L}$ for $\lambda$ inaccessible and $\lambda>\kappa$ with $R \leq \lambda$ should follow by the methods of Beller and Litman [6] but we have not checked this in detail.

7.4 Lemma. Suppose $2^{\mu}=\mu^{+}=\lambda, \mu^{\kappa}=\mu$, and $S, R,\left\langle C_{\delta}: \delta \in S\right\rangle$ have been chosen to satisfy all conditions of $\otimes_{\lambda, \kappa, R}(S)$ except ii) and vii). Suppose further that for each cub $E$ of $\lambda,\left\{\delta: \operatorname{acc}\left[C_{\delta}\right] \subseteq E\right\}$ is stationary in $\lambda$. Then $\otimes_{\lambda, \kappa, R}(S)$ holds.

\section{References}

[1] M. Karlowicz A. Engelking. Some theorems of set theory and their topological consequences. Fundamenta Mathematica, 57:275-285, 1965.

[2] M. Albert and R. Grossberg. Rich models. Journal of Symbolic Logic, 55:1292-1298, 1990.

[3] U. Avraham, S. Shelah, and R. Solovay. Squares with diamonds and Souslin trees with special squares. Fundamenta Mathematica, 127:133$162,1987$.

[4] J.T. Baldwin and S. Shelah. The primal framework: I. Annals of Pure and Applied Logic, 46:235-264, 1990.

[5] J.T. Baldwin and S. Shelah. The primal framework II: Smoothness. Annals of Pure and Applied Logic, 55:1-34, 1991. 
[6] A. Beller and A. Litman. A strengthening of Jensen's $\square$ principles. Journal of Symbolic Logic, 45:251-264, 1980.

[7] J. A. Makowsky. Abstract embedding relations. In J. Barwise and S. Feferman, editors, Model-Theoretic Logics, pages 747-792. SpringerVerlag, 1985.

[8] S. Shelah. Reflection of stationary sets and successor of singulars. preprint 351: to appear in Archive fur Mat. Log.

[9] S. Shelah. On the number of nonisomorphic models of cardinality $\lambda$ $L_{\infty \lambda}$-equivalent to a fixed model. Notre Dame Journal of Formal Logic, 22:5-10, 1981.

[10] S. Shelah. Models with second order properties IV, A general method and eliminating diamonds. Annals of Mathematical Logic, 38:183-212, 1983.

[11] S. Shelah. Remarks on squares. In Around Classification Theory of Models. Springer-Verlag, 1986. Springer Lecture Notes 1182.

[12] S. Shelah. Nonelementary classes II. In J. Baldwin, editor, Classification Theory, Chicago 1985. Springer-Verlag, 1987. Springer Lecture Notes 1292.

[13] S. Shelah. Universal classes: Part 1. In J. Baldwin, editor, Classification Theory, Chicago 1985, pages 264-419. Springer-Verlag, 1987. Springer Lecture Notes 1292. 